\title{
Knowledge and Perceptions of Teleaudiology Among Audiologists: A Systematic Review
}

\author{
Rohit Ravi ${ }^{1}$, Dhanshree R Gunjawate ${ }^{2}$, Krishna Yerraguntla ${ }^{1}$, and Carlie Driscoll ${ }^{3}$ \\ ${ }^{1}$ Department of Speech and Hearing, School of Allied Health Sciences, Manipal Academy of Higher Education, Manipal, Karnataka, \\ ${ }^{2}$ Department of Audiology and SLP, Kasturba Medical College, Mangalore, Manipal Academy of Higher Education, Manipal, Karnataka, \\ India \\ ${ }^{3}$ University of Queensland, School of Health \& Rehabilitation Sciences, Hearing Research Unit for Children, St Lucia, QLD, Australia
}

\author{
Received December 15, 2017 \\ Revised February 8, 2018 \\ Accepted March 12, 2018
}

Background and Objectives: The knowledge and perception of teleaudiology among audiologists will determine the acceptance and success of teleaudiology practice. This systematic review was conducted to review the published literature on knowledge and perceptions of teleaudiology application among audiologists. Materials and Methods: Five studies exploring knowledge and perceptions of teleaudiology application among audiologists published in the English language up to May 2017 were included. Results: Positive attitudes was observed across the studies regarding acceptance towards application of teleaudiology. The most common sources of knowledge were on the job, graduate studies, and continuing education programs. The major barriers to the uptake of application of teleaudiology were in terms of limitations in infrastructure, reimbursement, and licensure. Conclusions: The study sheds light on the existing knowledge and perceptions of teleaudiology applications among audiologists. This will help in improving the existing teleaudiology services as well as overcome the challenges faced.

J Audiol Otol 2018;22(3):120-127

KEY WORDS: Systematic-review · Telehealth · Audiologists.

\section{Introduction}

Telehealth aims to provide health care services to underserved populations across the world using communication and information technology. The word telehealth means health care at a distance [1]. Advanced technology and connectivity have helped in bridging the gap between patients and health care providers, thereby overcoming economic and geographical barriers. It has also enabled better access to professional training resources and mentoring for the health care professionals. Teleaudiology can be applied either in a synchronous, asynchronous or hybrid model. A synchronous model allows for real-time delivery and monitoring of services via videoconference. An asynchronous model uses the store-and-forward methodology, where the test results are stored and reviewed

This is an Open Access article distributed under the terms of the Creative Commons Attribution Non-Commercial License (http://creativecommons.org/licenses/by-nc/4.0/) which permits unrestricted non-commercial use, distribution, and reproduction in any medium, provided the original work is properly cited. later. The hybrid model uses both synchronous and asynchronous models [2].

The American Academy of Audiology [3] and American Speech-Language-Hearing Association [4] have propagated and supported the use of telehealth services through position statements. These services are especially applicable for those patients with limited access to healthcare resources, cost-efficient and can act as a supplement to face-to-face services [5]. Telehealth can be applied directly to patient care as well as indirectly for training health professionals [1]. Likewise, teleaudiology has found applications in hearing screening, diagnosis, intervention, and providing training/education to professionals $[6,7]$.

In the global scenario, there is a lack of educated, trained, and employed health workers for the number of people in need of medical services [8]. Audiological services help to provide early hearing detection, diagnosis, and intervention for individuals with hearing loss. A lack of adequate hearing health professionals has been noted in both developed and 
developing countries [6]. Thus, the application of teleaudiology helps in providing the audiological services along with an added advantage of serving the under-served and enhancing follow-up services.

A systematic review of telehealth applications in audiology shed light on sparse information available on patient and clinician perception regarding acceptance and use of telehealth applications. It further emphasized that teleaudiology holds a significant promise in helping to extend audiological services and the need to carry out empirical research [7]. Bush, et al. [9] reviewed the feasibility of implementing remote telehealth for providing auditory rehabilitation for users of hearing aids and cochlear implants. A paucity of literature was noted on cost-effectiveness, infrastructure, and reimbursement for longterm sustainability of these services. Another recent systematic review included 103 papers of which $32.1 \%$ focused on hearing [10]. Further, the majority focused on assessment (36.9\%) and intervention (36.9\%). The majority were published in the United States of America (32\%) and followed by Australia (29.1\%). Most studies concluded that telehealth has an advantage over face-to-face services. The review highlighted the need among audiologists to adapt to telehealth applications. These professionals should be familiar and aware about the use of telehealth for diagnostic and therapeutic purposes as well as for professional development activities [10].

With the advances in telecommunications, Internet services, and satellite communication it is now possible to focus on further development and advancement of the teleaudiology services. This can especially be beneficial in countries with poor service seeker to provider ratio. This expansion of services can be made possible with a thorough understanding of attitudes and perceptions of audiologists towards teleaudiology. Thus, the present systematic review aimed at exploring the existing literature on attitudes and perceptions of audiologists towards the teleaudiology. Further, it intended to ascertain the sources of their existing knowledge, and barriers to the successful uptake of teleaudiology.

\section{Materials and Methods}

The present systematic review was carried out using the Preferred Reporting Items for Systematic Reviews and Metaanalyses statement (PRISMA) guidelines [11]. It provides an evidence-based minimum set of items to conduct and report systematic reviews and meta-analyses.

\section{Search strategy for identification of studies}

Two researchers (RR and DG) independently carried out a systematic search across multiple electronic databases. The four electronic databases included were; PubMed/Medline, Scopus, Web of Science, and Cochrane Library. The main search terms included were 'audiologists,' 'practitioners,' 'telehealth,' 'teleaudiology,' 'telerehabilitation,' 'knowledge,' 'attitude,' 'perception,' 'views,' and 'opinions.' Boolean operators such as 'AND and 'OR' were used to create search strings for different databases. The search string used for PubMed/ Medline has been attached as Appendix as an example.

\section{Selection criteria}

A systematic search was carried out across the different electronic databases to identify studies exploring opinions or knowledge towards teleaudiology application among audiologists. Only cross-sectional or survey design studies published in the English language up to May 2017 were included. The hits from all the databases were compiled using Mendeley desktop reference management system, and duplicate studies were eliminated. The titles and abstracts were screened independently by two researchers for its suitability \& inclusion. A $100 \%$ agreement was noted among the authors for screening and shortlisting the articles. Full-texts of the final shortlisted articles were obtained for data extraction.

\section{Data extraction}

The data extraction was carried out using a predesigned form developed following a discussion among the authors. The form included aim, population details, study design, survey details, experience using teleaudiology, knowledge, knowledge resources, perceptions, barriers, and limitations.

\section{Methodological quality appraisal}

The methodological quality appraisal was carried out for included studies with yes/no based on six questions. The tool was developed based on standard guidelines for 'quality assessment tool for observational cohort and cross-sectional studies' [12].

\section{Results}

A total of 250 hits were obtained across the four electronic databases, of which 45 were duplicates and were eliminated. Titles of 205 studies were screened of which 199 studies were excluded, as they did not suit the inclusion criteria. Only studies exploring opinions or knowledge towards teleaudiology and its application among audiologists were included. Studies on patient perceptions towards teleaudiology and use of teleaudiology in telehealth were excluded. Abstracts of six studies were screened from which four studies were found suitable. The back references of the included four studies were 
screened to identify any additional studies and one study was found to be suitable for inclusion. Thus, five studies were included for data extraction. Fig. 1 depicts the PRISMA flow chart for the study search and retrieval process for included studies. Table 1 displays the study characteristics such as study

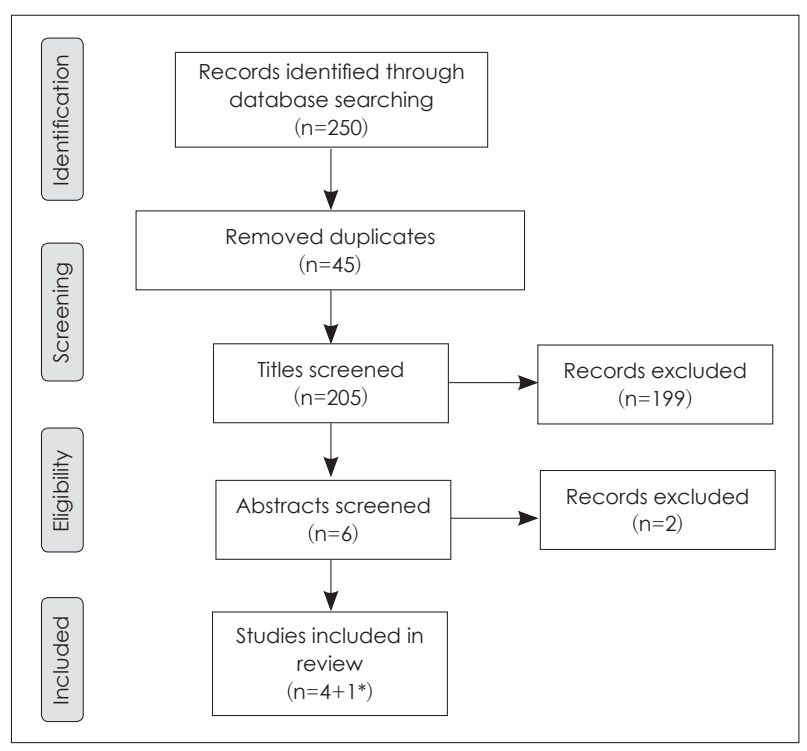

Fig. 1. PRISMA flowchart outlining the steps followed in the review. *added from back references of included articles. PRISMA: Preferred Reporting Items for Systematic Reviews and Meta-analyses statement. design, location, population, and questionnaire characteristics. Table 2 displays key data on experience and attitudes towards teleaudiology extracted from the five articles. Table 3 displays the methodological quality appraisal for the included studies.

\section{Sources and means to increase knowledge}

The most common source of knowledge of teleaudiology reported was on the job, graduate studies and continuing education through seminar/conference $[13,14]$. About $90 \%$ of the audiologists who already practiced teleaudiology were interested in increasing their knowledge and having additional training, while $75 \%$ of the audiologists who did not use telemode were interested in additional training to increase their knowledge. Across the respondents, the preferred means to get additional training were conferences, scientific literature, online courses, and websites [14].

\section{Barriers to the uptake of application of teleaudiology}

The review helped to ascertain barriers as reported by audiologists that restrict the application and use of teleaudiology. These included: lack of infrastructure and technology for the practitioner as well as the patient [13-15], lack of trained professionals [14,15], reimbursement issues, licensure laws, and confidentiality of patient information $[4,14]$. Some of the

Table 1. Characteristics of included studies

\begin{tabular}{|c|c|c|c|c|}
\hline Study ID & Location & Population & Study design & Questionnaire details \\
\hline ASHA [13] & USA & 842 audiologists & Telephone survey & $\begin{array}{l}\text { Demographics, ages served, } \\
\text { areas of practice/diagnosis, attitudes, } \\
\text { barriers to service delivery, expanding } \\
\text { telepractice, reimbursement, research } \\
\text { and technology, settings, } \\
\text { services delivered, training }\end{array}$ \\
\hline Schonfeld [14] & USA & $\begin{array}{l}422 \text { audiologists } \\
\text { (31 used teleaudiology, } \\
391 \text { not used) }\end{array}$ & Postal survey & $\begin{array}{l}\text { Demographics, clinical experience of users } \\
\text { and non-users of telehealth }\end{array}$ \\
\hline $\begin{array}{l}\text { Eikelboom } \\
\qquad \& \text { Swanepoel [15] }\end{array}$ & World & 269 audiologists & $\begin{array}{l}\text { Online survey } \\
\text { (using Limesurvey) }\end{array}$ & $\begin{array}{l}\text { Demographics, attitudes toward telehealth } \\
\text { working arrangements, education, and } \\
\text { use of technology }\end{array}$ \\
\hline Singh, et al. [24] & Canada & $\begin{array}{l}202 \text { audiologists and hear- } \\
\text { ing instrument specialists }\end{array}$ & $\begin{array}{l}\text { Online survey } \\
\text { (using LimeSurvey) }\end{array}$ & $\begin{array}{l}\text { Attitudes toward teleaudiology scale for } \\
\text { practitioners (ATS-P) 46-item survey in } \\
4 \text { sections; beliefs about teleaudiology } \\
\text { affecting different clinical and professional } \\
\text { practices, previous experience, and } \\
\text { willingness to conduct various clinical tasks } \\
\text { on various patient populations }\end{array}$ \\
\hline Dharmar, et al. [25] & USA & 12 audiologists & Observational & $\begin{array}{l}\text { Telehealth experience, overall experience } \\
\text { with teleaudiology, importance of clinical } \\
\text { service to be available over telehealth }\end{array}$ \\
\hline
\end{tabular}

ASHA: American Speech-Language-Hearing Association 


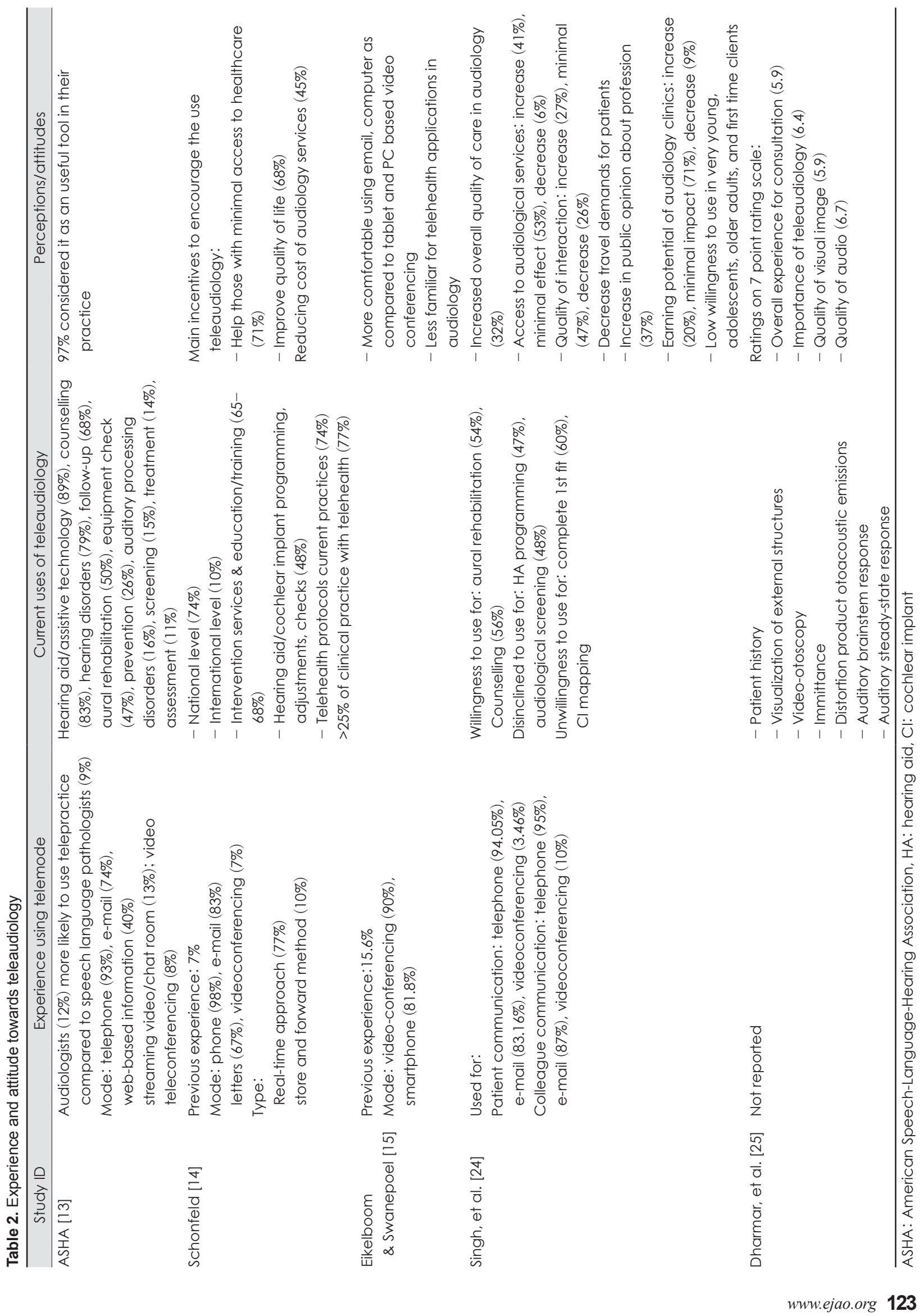


Table 3. Quality appraisal of studies

\begin{tabular}{lllllll}
\hline \multicolumn{1}{c}{ Study ID } & Q1 & Q2 & Q3 & Q4 & Q5 & Q6 \\
\hline ASHA [13] & Yes & No & Yes & Yes & No & No \\
Schonfeld [14] & Yes & Yes & Yes & Yes & No & No \\
Eikelboom \& Swanepoel [15] & No & Yes & Yes & Yes & No & No \\
Singh, et al. [24] & Yes & Yes & Yes & Yes & No & Yes \\
Dharmar, et al. [25] & No & Yes & Yes & Yes & No & No \\
\hline
\end{tabular}

Q1: Was the population well defined? Q2: Was the study objective clearly specified? Q3: Were the participants selected in unbiased and random manner? Q4: Was the survey unbiased? Q5: Was the sample size justification or power analysis carried out? Q6: Was the survey pilot tested or reviewed prior to the use?

additional concerns identified were: the reliability of results [14], cost, lack of standards, need for additional training/ knowledge, reduction in quality as compared to face-to-face interaction, and concern about malpractices [4].

\section{Discussion}

Telehealth services have been available since the late 19th century with the first published evidence emerging around 20th century where electrocardiography data was transmitted across telephone wires [16]. The recent growth in the application of telehealth can be attributed to growing internet use. Telehealth has made possible applications both within and across national borders [17]. In terms of application in audiology, studies have been carried out to report its application in domains of hearing screening, diagnosis, intervention and continuing education programs. The use of internet has become part and parcel of day to day life which can be evidenced by the increase in access to the internet from $16 \%$ of the world population in 2005 to $47 \%$ in 2016 [18]. The use of internet and technology has made provision of medical and rehabilitative services available even in rural and remote areas where there is a lack of access to care. These advancements in technology can be noted even in the field of teleaudiology with, within and across country applications. This widespread use of teleaudiology has enabled providing precise and reliable results, thereby further increasing its application [6,7,19-21].

The present study provides a systematic review of studies on knowledge and perceptions of teleaudiology application among audiologists. It further helps to identify the knowledge sources as well as barriers to the application of teleaudiology as reported by the audiologists themselves. Five studies were shortlisted after systematically reviewing the literature available. Three of these studies were carried out on audiologists from USA, one from Canada, and one across the world. Across all the studies, a total of 1,747 audiologists were surveyed on their knowledge and perception of teleaudiology application.

The provision of teleaudiology depends on several factors such as service providers/audiologists, infrastructure, highspeed internet, cost and socio-cultural issues. The success of service delivery of such program lies to a great extent on the service providers and their role. Further, in recent years there has been an added impetus towards increasing the applicability of teleaudiology. Therefore, in this context, it becomes crucial to have an understanding of the existing knowledge and perceptions of audiologists towards teleaudiology. The review findings have been discussed in terms of sources and means to increase knowledge, perceptions towards the application of teleaudiology, barriers to uptake of application of teleaudiology.

\section{Sources and means to increase knowledge}

The most common source of knowledge of teleaudiology reported was on the job, graduate studies, and continuing education through seminar/conference $[4,14]$. About $90 \%$ of the audiologists who already practiced teleaudiology were interested in increasing their knowledge and having additional training, while $75 \%$ of the audiologists who did not use the telemode were interested in additional training to increase their knowledge. This contrast can be because audiologists who already use teleaudiology are acquainted with its advantages and benefits. They might also be in a better position to compare their experience with teleaudiology to face-to-face interaction with the patients. Across the respondents, the preferred means to get additional training were conferences, scientific literature, online courses, and websites [14]. These are the sources were most updated information is available and hence could be a popular choice. Additional training sessions exclusively dealing with teleaudiology might be of benefit to the audiologists to keep themselves updated with the current trends and practices.

\section{Perceptions towards application of teleaudiology}

An overall positive trend was observed in the attitudes towards telehealth, which varied across the studies. These differences in attitudes could be due to varied experience and exposure in teleaudiology. Hanson, et al. [22] carried out a 
longitudinal study to explore the pre- and post- telehealth attitudes among health care providers to report the changes in attitude. First-time telehealth users exhibited more positive attitudes towards telehealth, with no changes in their attitude with subsequent use. This normalization of behavior as well its effect on attitude formation is often reported in the literature [23]. This could be the possible reason for the mixed responses obtained in the present review across studies.

\section{Barriers to the uptake of application of teleaudiology}

The barriers as reported by audiologists that restrict the application and use of teleaudiology included lack of facilities/infrastructure, training, reimbursement, the reliability of results, licensure, and reduced quality when compared to faceto-face interactions. This is similar to a study by Molini-Avejonas, et al. [10] which has identified barriers such as the need for software information, the speed of internet, and technological limitations that limit the use of telehealth in speech, language, and hearing sciences. Fabry [5] has pointed out issues in reimbursement, licensure, generation, and geographical barriers for application of telehealth.

\section{Further implications}

The field of teleaudiology based services is expanding in both developed and developing countries. The present systematic review helped to identify the attitudes, and perceptions of teleaudiology applications among audiologists. Based on the findings of this review, a need was felt to have future studies further to explore the knowledge, attitudes, and perceptions of audiologists, especially from developing countries. In this age of technological advancements, the studies should also explore the attitude, uptake, and acceptance of different types of technology.

Most of the studies are concentrated on developed countries where teleaudiology has a long history. Studies across both the developed and developing countries will enable a better understanding of the scope and acceptance of teleaudiology worldwide. Studies should also explore the acceptance of telemode by not just the professionals or providers but also the seekers or patients. The existing services can be improved to reach more people only with a thorough understanding of the available infrastructure, resources, software-hardware, as well as details of the cost-factor analysis. The present review will help in better planning for introducing teleaudiology services as well as improve service delivery.

\section{Limitations}

The included studies were mostly survey-based studies comprising of audiologists with and without experience with teleaudiology. The respondents were mostly from developed countries where such services have been available for several years. The heterogeneity of studies made the generalizations of results across the populations difficult. The review included studies published in the English language and studies in other languages, as well as unpublished grey literature, may have been missed.

\section{Conclusion}

The review sheds light on the existing knowledge and perceptions of teleaudiology applications among audiologists. Overall, positive attitudes was observed among audiologists towards applications of teleaudiology. The review also helped to identify the sources of knowledge gaps in the implementation of teleaudiology services. This will help in improving existing teleaudiology services as well as assist in overcoming some of the challenges faced.

\section{Conflicts of interest}

The authors have no financial conflicts of interest.

\section{REFERENCES}

1) Wootton R, Patil NG, Scott RE, Ho K, editors. Telehealth in the Developing World. London: Royal Society of Medicine Press Ltd;2009.

2) Krumm M. Audiology telemedicine. J Telemed Telecare 2007;13: 224-9.

3) American Academy of Audiology. The use of telehealth/telemedicine to provide audiology services [cited 2017 Aug 30]. Available from: http://audiology-web.s3.amazonaws.com/migrated/TelehealthResolution200806.pdf_5386d9f33fd359.83214228.pdf.

4) American Speech-Language-Hearing Association. Audiologists providing clinical services via telepractice: position statement. [cited 2017 Aug 30]. Available from: https://www.asha.org/policy/PS200500029.htm.

5) Fabry D. Applications of telehealth for hearing care. Audiol Today 2010;22:18-25.

6) Swanepoel de W, Clark JL, Koekemoer D, Hall JW 3rd, Krumm M, Ferrari DV, et al. Telehealth in audiology: the need and potential to reach underserved communities. Int J Audiol 2010;49:195-202.

7) Swanepoel de W, Hall JW 3rd. A systematic review of telehealth applications in audiology. Telemed J E Health 2010;16:181-200.

8) Global Health Workforce Alliance. Scaling up, saving lives: task force for scaling up education and training for health workers 2008 [cited 2017 May 23]. Available from: http://www.who.int/workforcealliance/documents/Global_Health\%20FINAL\%20REPORT.pdf.

9) Bush ML, Thompson R, Irungu C, Ayugi J. The role of telemedicine in auditory rehabilitation: a systematic review. Otol Neurotol 2016; 37:1466-74.

10) Molini-Avejonas DR, Rondon-Melo S, Amato CA, Samelli AG. A systematic review of the use of telehealth in speech, language and hearing sciences. J Telemed Telecare 2015;21:367-76.

11) Moher D, Liberati A, Tetzlaff J, Altman DG; PRISMA Group. Preferred reporting items for systematic reviews and meta-analyses: the PRISMA statement. PLoS Med 2009;6:e1000097.

12) NIH. Quality assessment tool for observational cohort and cross sectional studies. 2014 [cited 2017 May 23]. Available from: https:// www.nhlbi.nih.gov/health-topics/study-quality-assessment-tools. 
13) American Speech Language Hearing Association (ASHA). Survey of telepractice use among audiologists and speech-language pathologists. 2002 [cited 2017 May 23]. Available from: http://www.asha. org/uploadedFiles/practice/telepractice/SurveyofTelepractice.pdf.

14) Schonfeld YG. The use of telehealth by audiologists: a survey [dissertation]. Towson (MD): Towson Univ.;2016.

15) Eikelboom RH, Swanepoel W. International survey of audiologists' attitudes toward telehealth. Am J Audiol 2016;25:295-8.

16) World Health Organization. Telemedicine: opportunities and developments in member states. Global observatory for ehealth seriesvolume 2. World Health Organization;2010.

17) Poe K. Telemedicine liability: Texas and other states delve into the uncertainties of health care delivery via advanced communications technology. Rev Litig 2001;20:681-708.

18) International Telecommunication Union (ITU). ICT facts and figures 2016. Geneva: International Telecommunication Union (ITU);2016.

19) Elangovan S. Telehearing and the Internet. Semin Hear 2005;26:19-
25.

20) Givens GD, Elangovan S. Internet application to tele-audiology-"nothin' but net". Am J Audiol 2003;12:59-65.

21) Lancaster P, Krumm M, Ribera J, Klich R. Remote hearing screenings via telehealth in a rural elementary school. Am J Audiol 2008;17: 114-22.

22) Hanson D, Calhoun J, Smith D. Changes in provider attitudes toward telemedicine. Telemed J E Health 2009;15:39-43.

23) Ajzen I, Fishbein M. Understanding attitudes and predicting social behavior. Englewood Cliffs, NJ: Prentice-Hall;1980.

24) Singh G, Pichora-Fuller MK, Malkowski M, Boretzki M, Launer S. A survey of the attitudes of practitioners toward teleaudiology. Int $\mathrm{J}$ Audiol 2014;53:850-60.

25) Dharmar M, Simon A, Sadorra C, Friedland G, Sherwood J, Morrow $\mathrm{H}$, et al. Reducing loss to follow-up with tele-audiology diagnostic evaluations. Telemed J E Health 2016;22:159-64. 
Ravi R, et al.

\section{$\square$ Appendix}

\section{PubMed}

((((audiologist) OR practitioners)) AND (((telehealth) OR teleradiology) OR telerehabilitation)) AND (((((knowledge) OR attitude) OR perception) OR views) OR opinions) 